\title{
Best practice in thyroid pathology
}

\author{
C E Anderson, K M McLaren
}

J Clin Pathol 2003;56:401-405

Thyroid pathology is a specialist area but is often encountered by the general pathologist in a variety of forms including cytology, frozen sections, and resection specimens. In the thyroid gland, as for other endocrine organs, many aspects of diagnosis are unique to this area of histopathology; thus, the aims of this paper are to set out best practice guidelines which, although not entirely comprehensive, will be of practical use.

M ost pathology departments receive relatively small numbers of thyroid specimens compared with those from other organ systems, but they are common enough to be dealt with fairly frequently by the general pathologist. Therefore, every pathologist who reports thyroid specimens should have an understanding of the diagnostic peculiarities and pitfalls of thyroid cytology and histopathology.

\section{THE REQUEST FORM}

As for any specimen, the request form should be completed in full with patient details and clinical information. The information given should include the results of related biochemical and radiological investigations if applicable, such as thyroid stimulating hormone concentrations, anti-thyroid antibody titres, and ultrasound scan findings.

\section{FINE NEEDLE ASPIRATION}

The use of fine needle aspiration (FNA) in the evaluation of a thyroid nodule is a relatively noninvasive technique that can often be diagnostic and may prevent unwarranted surgery. The method of preparation used can give varying cytological appearances and each has advantages and disadvantages. Recently, newer techniques have been developed-for example liquid based cytology, which allow lysis of blood and the preparation of further samples or a cell block for immunocytochemistry. However, the cytological appearances with liquid based cytology are somewhat different to those on conventional smears Dr C E Anderson Department of Pathology, Royal Infirmary of Edinburgh, 51 Little France Crescent, Edinburgh EHI 6 4SA, UK

Catriona.Anderson@ed.ac.uk

Accepted for publication 15 January 2002

.................... and further experience of the technique is required. Indeed, one recent study has suggested that liquid based thin layer methods are not ideal for use in thyroid aspirates. ${ }^{1}$

\begin{abstract}
"The use of fine needle aspiration in the evaluation of a thyroid nodule is a relatively non-invasive technique that can often be diagnostic and may prevent unwarranted surgery"
\end{abstract}

Regardless of the technique used, an aspirate should only be regarded as adequate if at least six epithelial groups are present. It should be categorised into one of the following five diagnostic groups, as recommended in the forthcoming thyroid cancer minimum data set.

Thyl: non-diagnostic (either because of inadequate cellularity or if technical problems preclude diagnosis).

Thy2: non-neoplastic (features consistent with a multinodular goitre or thyroiditis).

Thy3: follicular lesions, including those where neoplasia cannot be definitely excluded. In particular, a cellular dominant nodule is indistinguishable from a follicular neoplasm and the lesion should be managed as such.

Thy4: suspicious of malignancy.

Thy5: diagnostic of malignancy with unequivocal features of papillary (fig 1), medullary, or anaplastic carcinoma, or of lymphoma or metastasis.

In the case of an aspirate that is predominantly composed of lymphocytes, a diagnosis of Hashimoto's thyroiditis is supported by the presence of three components; namely, lymphocytes, plasma cells, and oncocytic epithelium, in conjunction with an appropriate clinical history.

\section{THYROID FROZEN SECTIONS}

There is still debate concerning the best operative procedure for differentiated thyroid carcinoma. In several centres, lobectomy is chosen for the dominant nodule in multinodular goitre, follicular adenoma, and minimally invasive follicular carcinoma, with total thyroidectomy reserved for widely invasive follicular carcinoma and papillary carcinoma. In some centres, all differentiated thyroid carcinomas are managed by total thyroidectomy. Conversely, a microfocus of papillary carcinoma or an intracystic papillary carcinoma may simply justify long term follow up rather than

Abbreviations: CK19, cytokeratin 19; FNA, fine needle aspiration 


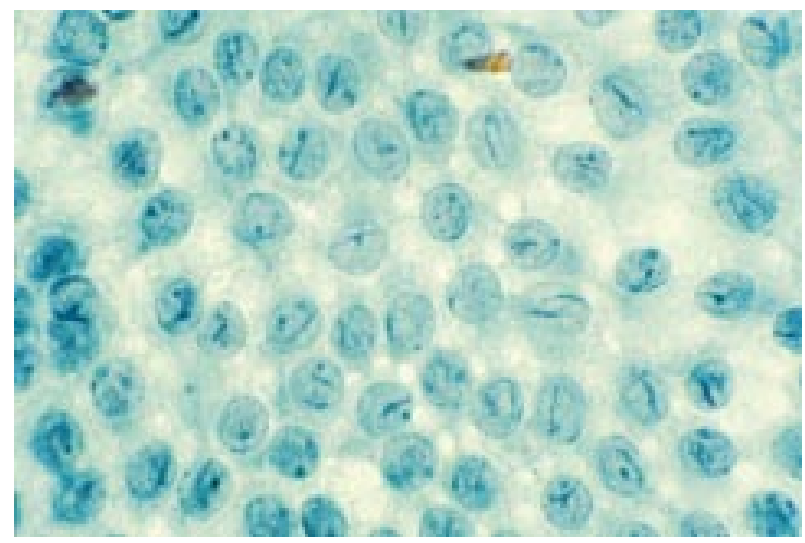

Figure 1 Fine needle aspirate of a papillary carcinoma showing nuclear grooves and optical clarity (Papanicolaou stain).

immediate radical surgery. Disagreement also exists over the use of frozen section in the intraoperative assessment of thyroid nodules.

The guidelines of the British Society of Endocrine Surgeons state that a preoperative FNA is desirable in the assessment of every solitary thyroid nodule, ${ }^{2}$ and if this is diagnostic of papillary carcinoma, then it is generally agreed that a frozen section is unnecessary. ${ }^{3}$ If FNA was not performed or the result was inadequate or only suspicious of papillary carcinoma then a request for a frozen section may result.

The main arguments against performing frozen section are first the cytological appearances, with potentially misleading nuclear optical clarity and difficulty in discerning nuclear grooves and intranuclear protrusions. Second, dissection of the soft, freshly removed gland inevitably causes architectural disruption that can make evaluation of the capsule difficult. Third, a diagnosis of minimally invasive follicular carcinoma is unlikely to be made on a frozen section because this would require the identification of focal capsular or vascular invasion, which may not be present in the section taken. However, in cases where no preoperative diagnosis is available, many surgeons will request a frozen section and each department should come to an agreement on this matter with the local surgical team, perhaps after determining local reliability of FNA and frozen sections. ${ }^{4}$

In our department, we perform frozen sections on solitary thyroid nodules in some circumstances and we follow a protocol that seeks to minimise the problems listed above (fig 2). After routine measurements, the thyroid capsule is inspected

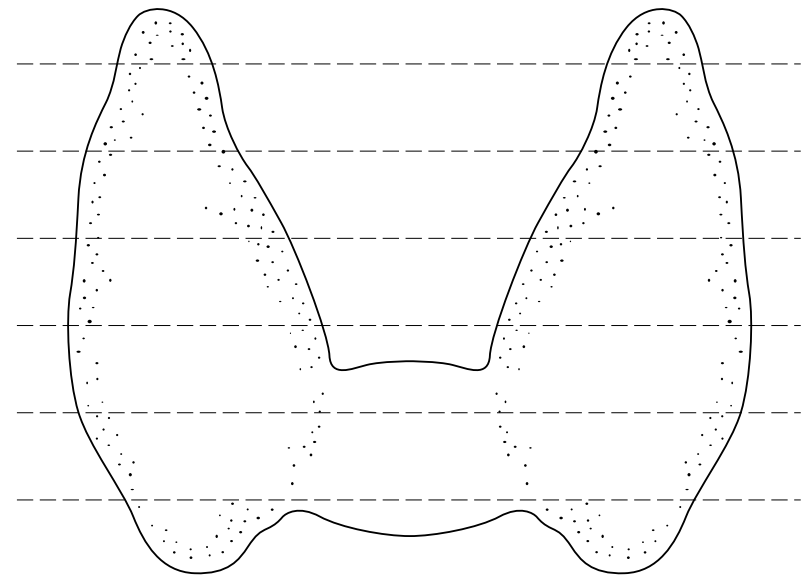

Figure 3 Where possible, the thyroid gland should be sectioned horizontally as demonstrated above.

and any palpable lesion measured, before taking one complete transection through the lesion. This enables the assessment of encapsulation, solidity, and the degree of cystic change. Although not widely used, a dab imprint of the cut surface is diagnostically valuable, ${ }^{5}$ and the cytological information so obtained is helpful in distinguishing a papillary carcinoma from a follicular neoplasm. If it suggests papillary carcinoma, then one confirmatory frozen section is performed. If it suggests a follicular neoplasm, then the specimen should be fixed for subsequent dissection, as described below. In this way, a papillary carcinoma may be diagnosed with confidence and a widely invasive follicular carcinoma distinguished from the benign or minimally invasive counterparts purely on macroscopic inspection accompanied by cytological affirmation that it is a follicular lesion.

\section{GROSS EXAMINATION}

\section{Thyroid tumours}

The specimen should be described as a total thyroidectomy, left lobectomy, or right lobectomy ( \pm isthmus). The specimen should be weighed, measured, and then the external appearance described, paying particular attention to whether the capsule is intact. For a total thyroidectomy specimen each lobe should be measured separately.

In most cases, the specimen should then be sectioned horizontally (fig 3). In the case of a probable follicular neoplasm, one author has put forward an alternative way of sectioning in an effort to optimise sampling of the capsule. ${ }^{6}$ In this method,

FNA - follicular lesion $\longrightarrow$ Send specimen in fixative

Figure 2 Possible protocol for dealing with thyroid specimens. FNA, fine needle aspiration; FS, frozen section. 
Table 1 Information to be noted in malignant thyroid tumours

- Single site or multifocal

- Completeness of excision

- Presence of extension beyond thyroid

- Presence of lymphovascular invasion

- Site and number of involved nodes Table 2 Classic nucl
papillary carcinoma

- Nuclear optical clarity

- Nuclear grooves

- Sharply delineated nuclear membranes

- Intranuclear cytoplasmic protrusions

- Nuclear overlapping

follicular neoplasms are bisected in the centre and then each half incised at intervals of $2-3 \mathrm{~mm}$ in the capsular plane. Each cut should not be completed until the whole lesion has been incised and then the cuts can be continued to free each slice in turn. This method avoids tangential cutting of the capsule and the difficulty encountered when only a small piece of tissue is left to cut.

The site of the tumour should be noted, along with the distance to the nearest excision margin. The size of the lesion must be noted for the pT aspect of pathological staging, taking the size of the largest lesion if more than one is identified. Extension of the tumour beyond the thyroid gland should be noted.

"In papillary carcinoma, the tumour type should be specifically mentioned, because the tall cell, oncocytic, solid, and diffuse sclerosing variants are said to have a worse prognosis than papillary carcinoma of the classic type"

Representative blocks should be selected based on the probable histological nature of the lesion. It is well recognised that papillary carcinoma can be multifocal ${ }^{7}$ and, therefore, if this diagnosis is suspected, the adjacent and opposite lobe should be sampled and any pale areas processed. In a follicular lesion that is not grossly invasive, the interface between the tumour and the capsule and the tumour and the adjacent gland

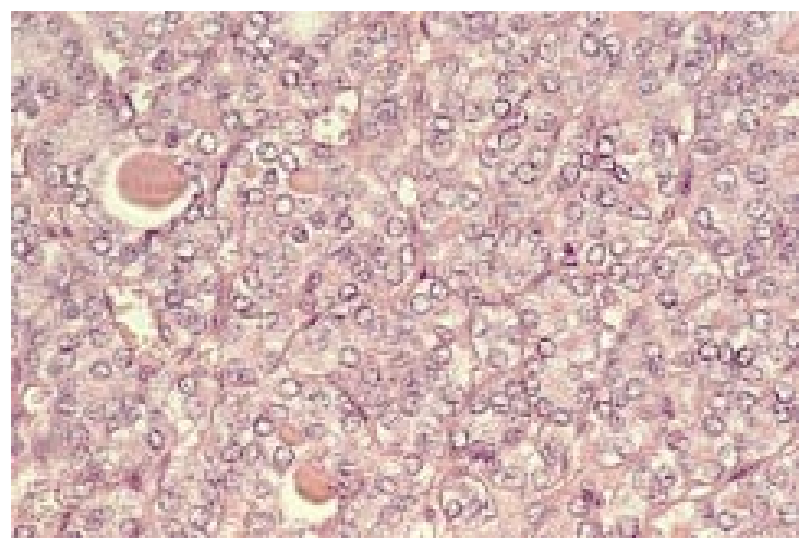

Figure 4 Follicular variant of papillary carcinoma (haematoxylin and eosin stain). should be extensively sampled to look for capsular and vascular invasion. In large lesions, at least 10 blocks should be taken and small lesions should be processed in their entirety. In medullary carcinoma, the upper part of the lobes should be sampled because the proportion of $\mathrm{C}$ cells is greater in this area, and therefore $\mathrm{C}$ cell hyperplasia is more likely to be detected.

Any lymph nodes submitted with the specimen should be dissected in the usual way, noting whether they are ipsilateral or contralateral to the tumour, or in the midline.

\section{Non-neoplastic thyroids}

These specimens should be described, weighed, and sectioned as above. The slices should be carefully examined for the presence of pale foci because incidental carcinomas can be found in Hashimoto's thyroiditis, multinodular goitre, and Graves' disease. $^{8-10}$

\section{MICROSCOPIC EXAMINATION \\ Thyroid tumours}

Treatment and prognosis are closely related to histological tumour type and accurate staging, ${ }^{11}$ and therefore a thorough microscopic examination is essential. For malignant tumours, the information listed in table 1 should be noted and the TNM staging should be recorded.

The diagnosis of papillary carcinoma is based on the identification of true papillary architecture in the classic type, in addition to several well recognised nuclear features (table 2). In particular, care should be taken to assess the nuclear morphology of apparently follicular lesions so that the follicular variant of papillary carcinoma is not overlooked. Clues helpful in identifying the follicular variant (fig 4) include intensely staining colloid with a scalloped outline, ${ }^{12}$ and multinucleate giant cells within the follicles. A recent study has described further criteria including the presence of cells with dense, cerebriform nuclei and overall distortion of the follicular architecture. ${ }^{13}$ In papillary carcinoma, the tumour type should be specifically mentioned, because the tall cell, solid, and diffuse sclerosing variants are said to have a worse prognosis than papillary carcinoma of the classic type..$^{14}$

Malignant follicular neoplasms should be described as either widely or minimally invasive. A widely invasive follicular carcinoma shows macroscopic invasion or extensive infiltration of the surrounding thyroid parenchyma and vessels microscopically. In contrast, a minimally invasive follicular neoplasm, which has an excellent long term prognosis, is defined as that which shows capsular invasion or invasion of vessels within or adjacent to the capsule. ${ }^{16}$ In this regard, the presence of capsular thickening, even focally, is a suspicious feature that should prompt a careful search for

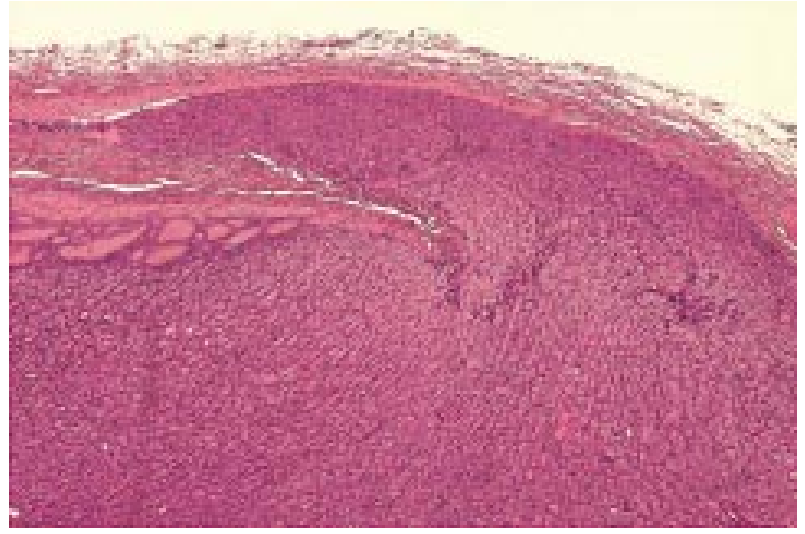

Figure 5 Minimally invasive follicular carcinoma-the invasive tongue of tumour has completely penetrated the capsule of the neoplasm (haematoxylin and eosin stain). 


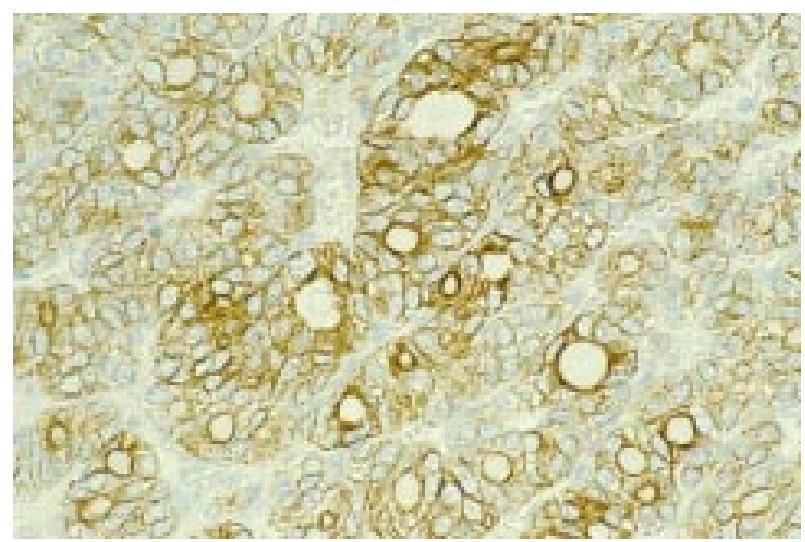

Figure 6 Immunohistochemistry for cytokeratin 19 in the follicular variant of papillary carcinoma.

capsular or vascular invasion. Capsular invasion is defined as complete penetration of the capsule by an invasive tongue of tumour (fig 5) and should not be confused with entrapment of follicles or pseudoinvasion as a result of previous FNA. This last situation is usually associated with inflammation and haemosiderin deposition.

In a case of medullary carcinoma, it is conventional to describe the cellular pattern of the lesion, although this has no known prognostic relevance. The presence of amyloid should be noted because this is thought to be associated with a better prognosis. ${ }^{17}$ Confirmation of the nature of the carcinoma by immunocytochemistry for calcitonin and carcinoembryonic antigen is usual, particularly in poorly differentiated lesions, although immunoreactivity for calcitonin may be lost in these cases. All newly diagnosed medullary carcinomas should now be offered genetic testing because of the association with multiple endocrine neoplasia syndromes.

If a tumour is undifferentiated, immunocytochemistry for thyroglobulin and calcitonin should be performed in an attempt to identify a differentiated component. In cases lacking such hormonal expression, cytokeratin markers may at least permit distinction from a sarcoma, and crucially in a lesion of small cell type, from a lymphoma. If lymphoma involving the thyroid is suspected then the appropriate immunocytochemical markers should be selected.

\section{Immunocytochemistry in solitary thyroid nodules}

The role of immunocytochemistry in the diagnosis of solitary thyroid nodules has developed considerably over the past few years. Several studies have shown that cytokeratin 19 (CK19) is intensely expressed by almost all examples of papillary carcinoma. ${ }^{18-20}$ Its value lies in the distinction between papillary carcinoma and papillary hyperplasia-for example, in a follicular adenoma-although this is compounded somewhat by the finding of focal weak CK19 positivity in some follicular adenomas. ${ }^{21}$ Immunocytochemistry for S100 can also be of use because it is typically expressed by papillary carcinomas but not by areas of papillary hyperplasia. ${ }^{22}$ The difficulty of an encapsulated nodule showing a follicular growth pattern and focal areas with some but not all of the cytological features of papillary carcinoma is well recognised, and CK19 positivity in these cases lends weight to a diagnosis of the follicular variant of papillary carcinoma ${ }^{24}$ (fig 6).

Galectin 3 is a carbohydrate binding lectin that appears to play a role in diverse processes such as embryogenesis, inflammation, apoptosis, and neoplasia. ${ }^{26}$ It is expressed in a high proportion of thyroid papillary and follicular carcinomas but is seldom expressed, other than in scattered cells, in follicular adenomas or dominant nodules of multinodular goitre. ${ }^{2027}$ If the various reports describing this pattern are

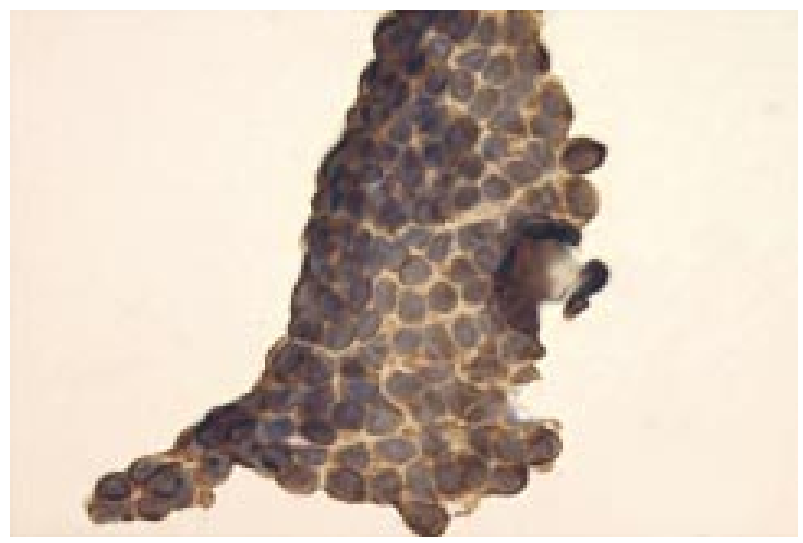

Figure 7 Immunohistochemistry for cytokeratin 19 in a fine needle aspirate of papillary carcinoma (Giemsa preparation with immunohistochemistry performed after removal of the coverslip).

borne out, this antibody may be of particular value in distinguishing a minimally invasive carcinoma from a cellular follicular adenoma, because galectin 3 positivity would prompt further sampling of the lesion to identify foci of capsular invasion.

The use of CK19 and galectin 3 could potentially be extended to augment the interpretation of a preoperative FNA. The cytological features of a papillary carcinoma may on occasion be focal or subtle, and the expression of CK19 with or without galectin 3 would add weight to the impression (fig 7). The absence or presence of galectin 3 may supplement the cytological features of a follicular neoplasm, with a negative result supporting a benign conclusion.

Obviously, a diagnosis of papillary carcinoma requires the correct nuclear criteria and minimally invasive follicular carcinoma, the correct architectural features. The pattern of expression of CK19 and galectin 3 should therefore be used to supplement the histological impression. A further caveat with the use of these antibodies is that both can be positive in nonneoplastic thyroid epithelium in the context of inflammation. ${ }^{29}$ Therefore, it remains to be seen how useful these markers really become in routine practice.

"All newly diagnosed medullary carcinomas should
now be offered genetic testing because of the
association with multiple endocrine neoplasia
syndromes"

In the assessment of papillary lesions, immunohistochemistry for the ret protooncogene product can also be helpful in identifying papillary carcinomas, although not all papillary carcinomas will be positive and findings vary between the histological types. ${ }^{30}$

\section{Non-neoplastic thyroid disease}

In the microscopic assessment of non-neoplastic thyroid disease, in addition to correctly diagnosing the non-neoplastic process involving the gland, care must be taken to exclude any foci of incidental malignancy. Obviously, any areas that were macroscopically suspicious should be examined carefully. In Hashimoto's thyroiditis, the diagnosis of an associated lymphoma can be particularly troublesome. Areas showing complete filling and expansion of follicles by lymphocytes or complete loss of epithelium are worrying, and immunocytochemistry or in situ hybridisation for light chain restriction may be helpful. However, the use of the polymerase chain reaction to detect a monoclonal gene rearrangement is the preferred method for confirmation of subtle changes in lesions lacking sufficient histological evidence of lymphoma. 


\section{Take home messages}

- Thyroid fine needle aspiration is an effective diagnostic tool and should be performed preoperatively in all solitary nodules

- The use of thyroid frozen section is disputed among both pathologists and surgeons; as yet, there is no consensus and this issue should be discussed with the multidisciplinary team at a local level

- In a follicular neoplasm, the capsule should be extensively sampled to identify any foci of capsular or vascular microinvasion

- In the microscopic examination of follicular lesions, the follicular variant of papillary carcinoma should be considered

- Several new immunohistochemical markers are reported to be helpful in the diagnosis of solitary nodules, but their use in routine practice is still being evaluated

\section{CONCLUSION}

The pathology of the thyroid gland presents the pathologist with a particular set of diagnostic problems. If best practice and the minimum data set guidelines are adhered to, the correct diagnosis should be reached in most cases. Newer techniques such as immunocytochemistry can certainly be helpful in more difficult cases but, as in all areas of pathology, histological features take precedence and good communication with the relevant clinical colleagues is paramount.

\section{Authors' affiliations}

C E Anderson, K M McLaren, Department of Pathology, Royal Infirmary of Edinburgh, 51 Little France Crescent, Edinburgh EH16 4SA, UK

\section{REFERENCES}

1 Nasuti JF, Tam D, Gupta PK. Diagnostic value of liquid-based (Thinprep) preparations in nongynecologic cases. Diagn Cytopathol 2001;24:137-41

2 The British Association of Endocrine Surgeons. Guidelines for the surgical management of endocrine disease. London: The British Association of Endocrine Surgeons, 2000

3 Chen $\mathrm{H}$, Zeiger MA, Clark DP, et al. Papillary carcinoma of the thyroid: can operative management be solely based on fine-needle aspiration? J Am Coll Surg 1997; 184:605-10.

4 Hamburger $\mathrm{JI}$, Husain M. Contribution of intraoperative pathology evaluation to surgical management of thyroid nodules. Endocrinol Metab Clin North Am 1990; 19:509-22.

5 Taneri F, Poyraz A, Salman B, et al. Using imprint and frozen sections in determining the surgical strategies for thyroid pathologies. Endocr Regul 2001;35:71-4

6 Yamashina M. Follicular neoplasms of the thyroid. Total circumferential evaluation of the fibrous capsule. Am J Surg Pathol 1992;16:392-400.

7 Pacini F, Alisei R, Capezzone $M$, et al. Contralateral papillary thyroid cancer is frequent at completion thyroidectomy with no difference in low and high risk patients. Thyroid 2001;11:877-81.

8 Eisenberg BL, Hensley SD. Thyroid cancer with coexistent Hashimoto's thyroiditis. Clinical assessment and management. Arch Surg

1989:124:1045-7.
9 Saiid MA Anwar M, Magbool M. Incidence of carcinoma in nodular goitre. Medical Monthly Forum 2000;1 1:5-6.

10 Farbota LM, Calandra DB, Lawrence AM, et al. Thyroid carcinoma in Graves' disease. Surgery 1985;98:1148-53.

11 Loh KC, Greenspan FS, Gee L, et al. Pathological tumour-node-metastasis (pTNM) staging for papillary and follicular thyroid carcinomas: a retrospective analysis of 700 patients. J Clin Endocrinol Metab 1997;82:3553-62.

12 Rosai J, Zampi G, Carcangiu ML. Papillary carcinoma of the thyroid. Am J Surg Pathol 1983;7:809-17.

13 Bell CD, Coire C, Treger T, et al. The "dark nucleus" and disruptions of the follicular architecture: possible new histological aids for the diagnosis of the follicular variant of papillary carcinoma of the thyroid. Histopathology 2001;39:33-42.

14 Moreno Egea A, Rodriguez Gonzalez JM, Sola Perez J, et al. Clinicopathological study of the diffuse sclerosing variety of papillary carcinoma of the thyroid. Presentation of four new cases and a review of the literature. Eur J Surg Oncol 1994;20:7-11.

15 Nikiforov YE, Erickson LA, Nikiforova MN, et al. Solid variant of papillary thyroid carcinoma: incidence, clinical-pathologic characteristics, molecular analysis and biologic behaviour. Am J Surg Pathol 2001;25:1478-84

16 Thompson LD, Wieneke JA, Paal E, et al. A clincopathologic study of minimally invasive follicular carcinoma of the thyroid gland with a review of the English literature. Cancer 2001;91:505-24.

17 Scopsi L, Sampietro G, Borracchi P, et al. Multivariate analysis of prognostic factors in sporadic medullary carcinoma of the thyroid. A retrospective study of 109 consecutive patients. Cancer 1996;78:2173-83.

18 Miettinen M, Kovatich AJ, Karkkainen P. Keratin subsets in papillary and follicular thyroid lesions. A paraffin section analysis with diagnostic implications. Virchows Arch 1997;431:407-13.

19 Raphael SJ, McKeown-Eyssen G, Asa SL, et al. High-molecular-weight cytokeratin and cytokeratin-19 in the diagnosis of thyroid tumours. Mod Pathol 1994;7:295-300.

20 Beesley MF, McLaren KM. Cytokeratin 19 and galectin-3 immunohistochemistry in the differential diagnosis of solitary thyroid nodules. Histopathology 2002;41:236-43.

21 Sahoo S, Hoda SA, Rosai J, et al. Cytokeratin 19 immunoreactivity in the diagnosis of papillary thyroid carcinoma: a note of caution. Am J Clin Pathol 2001;116:696-702.

22 Kilicarslan B, Pesterelli EH, Oren N, et al. Epithelial membrane antigen and S-100 protein expression in benign and malignant papillary thyroid neoplasms. Adv Clin Pathol 2000:4:155-8.

23 McLaren KM, Cossar DW. The immunohistochemical localization of $\mathrm{S} 100$ in the diagnosis of papillary carcinoma of the thyroid. Hum Pathol 1996;27:633-6.

24 Baloch ZW, Abraham S, Roberts S, et al. Differential expression of cytokeratins in follicular variant of papillary thyroid carcinoma: an immunohistochemical study and its diagnostic utility. Hum Pathol 1999;30:1 166-71.

25 Stephenson TJ. Papillary carcinoma of the thyroid: a tumour still with no benign neoplastic counterpart. Histopathology 2001;39:536-8.

26 Rabinovich GA, Baum LG, Tinari N, et al. Galectins and their ligands: amplifiers, silencers or tuners of the inflammatory response? Trends Immunol 2002;23:313-20.

27 Xu XC, el-Naggar AK, Lotan R. Differential expression of galectin-1 and galectin-3 in thyroid tumours; potential diagnostic implications. Am J Pathol 1995;147:815-21.

28 Inohara H, Honjo Y, Yoshii T, et al. Expression of galectin-3 in fine needle aspirates as a diagnostic marker differentiating benign from malignant thyroid neoplasms. Cancer 1999;85:2475-84.

29 Fernandez PL, Merino M, Gomez M et al. Galectin-3 and laminin expression in neoplastic and non-neoplastic thyroid tissue. J Pathol 1997; 181:80-6.

30 Cheung CC, Ezzat SE, Freeman JL, et al. Immunohistochemical diagnosis of papillary thyroid carcinoma. Mod Pathol 2001;14:338-42. 